TEMAS DE

ACTUALIDAD

Rev Chil Salud Pública 2018,

Vol 22(2): 171-178
PATRICIA CASTAÑEDA

Licenciada en Trabajo Social. Doctora en Ciencias de la Educación Escuela de Trabajo Social, Universidad de Valparaíso patricia.castaneda@uv.cl

KETTY CAZORLA Licenciada en Trabajo Social, Magister en Salud Pública Escuela de Trabajo Social, Universidad de Valparaíso

KATHERINE CUEVAS

Licenciada en Trabajo Social, Magister en Salud Pública

Departamento de Salud Pública, Escuela de Medicina, Universidad de Valparaíso Articulo recibido el 06/06/2018 Aceptado el 09/10/2018

\section{A 40 AÑOS DE LA DECLARACIÓN DE ALMA ATA. SALUD PARA TODOS EN LA ATENCIÓN PRIMARIA CHILENA}

\author{
To 40 years of declaration of Alma ATA. Health for all \\ IN CHILEAN PRIMARY CARE
}

\section{INTRODUCCIÓN}

En 1978 se celebra en Alma-Ata URSS, la Conferencia Internacional sobre Atención Primaria de Salud, organizada por la Organización Mundial de la Salud OMS y el Fondo de las Naciones Unidas para la Infancia UNICEF. Su declaración final plantea como meta la Salud para Todos en el año 2000, aportando una renovada definición para la atención primaria, transformándola desde un nivel de atención formal a una estrategia de desarrollo factible de implementar en todas las regiones del mundo. La declaración define en su Artículo VI a la atención primaria de salud en los siguientes términos:

"Es la asistencia sanitaria esencial basada en métodos y tecnologías prácticos, científicamente fundados y socialmente aceptables, puesta al alcance de todos los individuos y familias de la comunidad mediante su plena participación y a un costo que la comunidad y el país puedan soportar, en todas y cada una de las etapas de su desarrollo con un espíritu de autorresponsabilidad y autodeterminación. La atención primaria forma parte integrante tanto del sistema nacional de salud, del que constituye la función central y el núcleo principal, como del desarrollo social y económico global de la comunidad. Representa el primer nivel de contacto de los individuos, la familia y la comunidad con el sistema nacional de salud, llevando lo más cerca posible la atención de salud al lugar donde residen y trabajan las personas, y constituye el primer elemento de un proceso permanente de asistencia sanitaria". ${ }^{1}$

A 40 años de la Conferencia, el presente artículo revisa la trayectoria de la atención primaria chilena durante el período 1978-2018, a partir de los criterios de universalidad, participación en salud e intersectorialidad, referentes que sintetizan los principales planteamientos de Alma Ata.

Artículo no posee fuente de apoyo económico.

No existen conflictos de intereses. 
Trayectoria de los criterios propuestos por Alma Ata en la Atención Primaria de Salud chilena

1. Universalidad. Busca reducir inequidades y aumentar la capacidad resolutiva de la atención primaria en poblaciones usuarias y territorios focalizados. En 1978, Chile contaba con una red pública que integraba verticalmente las funciones de financiamiento y provisión de servicios sanitarios en el país ${ }^{2}$. Esta situación fue modificada estructuralmente por la dictadura chilena a partir de año 1979, a través del traspaso a las municipalidades de los activos, recursos financieros, dotación y normas de administración financiera de las estaciones médico rurales, postas rurales y consultorios generales urbanos y rurales, manteniendo el Ministerio de Salud la supervisión técnica. ${ }^{3}$ Esta situación fragilizó el financiamiento de la atención primaria, el que se realizó por una modalidad de pagos basada en la facturación por atención prestada en establecimientos municipalizados, conocida por su sigla FAPEM. El arancel estaba determinado por el tipo de acciones realizadas, valorizando las acciones curativo-asistenciales por sobre la promoción, prevención y educación en salud. ${ }^{4}$ El monto total de cada establecimiento estaba predefinido, sin hacerse cargo de las acciones excedentes, desfinanciando progresivamente el sistema y provocando serios déficit en inversiones de personal, infraestructura, tecnología, insumos, transportes y comunicaciones. En este marco, originalmente se esperaba contar con aportes complementarios de la población usuaria en lógica de copago y con recursos provenientes de los municipios. La administración municipal enfrentaba su propio déficit dadas las sucesivas crisis económicas de la década de 1980, generando un escenario adverso que ligó la realidad socioeconómica de cada comuna a la cantidad y calidad de servicios que podía ofrecer en atención primaria, situación especialmente crítica en los municipios que concentraban poblaciones de bajos ingresos, con alta demanda por salud municipal. ${ }^{5}$

Esta situación persistió hasta inicios de la transición democrática, en que las nuevas autoridades sectoriales realizaron importantes esfuerzos para compensar el déficit histórico.
El primer gesto reparatorio ocurrió en marzo de 1990, al restituir la gratuidad de las prestaciones de salud primaria a la población beneficiaria del Fondo Nacional de Salud en todos sus tramos. Junto con ello, se priorizaron las áreas de financiamiento, administración y cobertura y se organizó el Departamento de Atención Primaria del Ministerio de Salud. En el año 1994 se redefinió el financiamiento por el sistema per cápita, mejorando la equidad en la asignación de recursos y generando incentivos a la provisión y calidad de las prestaciones ofrecidas. Para fortalecer la capacidad resolutiva, se implementaron Servicios de Atención Primaria de Urgencia SAPU, asegurando atención médica de urgencia de baja complejidad en horarios no hábiles, incrementando la resolutividad local. ${ }^{6}$ A contar del año 1994 se implementaron los Servicios de Atención Médica de Urgencia SAMU, para entregar primera respuesta pre hospitalaria frente a emergencias médicas en forma integrada con la atención primaria. ${ }^{7}$ En forma complementaria, se desarrollaron Servicios de Urgencia Rural SUR adscritos a los Consultorios Generales Rurales, para introducir criterios de equidad en las comunas rurales más pobres del país, financiando la atención de urgencia fuera del horario hábil, aumentando accesibilidad, disponibilidad, eficiencia e impacto en las acciones de salud frente a demandas impostergables de atención médica. $^{8}$ En el año 1995 se promulgó el Estatuto de Atención Primaria, ${ }^{9}$ normando su administración, financiamiento y coordinación desde los principios de descentralización y desconcentración. Además, el cuerpo legal reguló la relación laboral, la carrera funcionaria y los deberes y derechos del personal, fuertemente afectados con el proceso de municipalización. También en la década de 1990 se implementaron salas de Infecciones Respiratorias Agudas (IRA), para atención prioritaria de la población infantil, oferta que posteriormente se amplió a las Salas de Atención de Enfermedades Respiratorias en población Adulta o Salas ERA. En la misma década, se incorporaron programas destinados a la atención de la salud mental de la población, enriqueciendo progresivamente su quehacer a través de la creación de los Centros Comunitarios de Salud Mental, COSAM. ${ }^{10}$ 
El año 2004, en el marco de la Reforma de Salud, se promulgó la Ley $N^{\circ} 19.996$ que estableció un Régimen General de Garantías en Salud, ${ }^{11}$ instrumento de regulación sanitaria que definió las prestaciones promocionales, preventivas, curativas, de rehabilitación y paliativas, junto con los programas que el Fondo Nacional de Salud debe cubrir en su población beneficiaria en su modalidad de atención institucional, siendo definida la atención primaria como la puerta de entrada al sistema. El Régimen General de Garantías incluye las Garantías Explícitas en Salud GES relativas a acceso, calidad, protección financiera y oportunidad de las prestaciones asociadas a un conjunto priorizado de programas, enfermedades o condiciones de salud. Asimismo, la Reforma de Salud impulsa a contar del año 2005 el Modelo de Atención Integral en Salud Familiar y Comunitaria, ${ }^{12}$ definiendo a la población usuaria en el centro del quehacer sanitario y como eje del desarrollo social. Se transforman progresivamente los establecimientos de atención primaria a Centros de Salud Familiar y Comunitaria CESFAM, focalizando territorios y poblaciones específicas a través de los Centros Comunitarios de Salud Familiar CECOSF. A contar del año 2006 se implementó la Política de Salud y Pueblos Indígenas, aportando con un enfoque de salud intercultural enriquecedor del quehacer de la atención primaria. ${ }^{13}$ Finalmente, en la búsqueda de mejor resolutividad conforme a los recursos locales disponibles, se refieren experiencias de contratación de profesionales especialistas en equipos de atención primaria. $\mathrm{Si}$ bien los esfuerzos desplegados pudieran resultar aún insuficientes, se constatan avances que incorporan componentes de accesibilidad, cobertura, financiamiento y calidad de servicios que contribuyen con la meta de Salud para Todos declarada por Alma Ata en 1978.

2. Participación en Salud. Alma Ata la concibe como componente central e irrenunciable en la estrategia que busca garantizar salud para todos, instando a superar modalidades consultivas o meramente informativas. La declaración valora la participación individual y colectiva en la planificación y aplicación de la atención de salud, relevando la importancia de comprender el proceso de salud-enfermedad desde una perspectiva que asciende en complejidad en relación a la calidad de la participación esperada. Al promover mayor empoderamiento individual y colectivo de la población usuaria, la salud y la enfermedad son entendidas como circunstancias de la esfera privada de las personas que impactan en la esfera colectiva, debiendo ser asumidas con responsabilidad pública.

En 1978, la participación estaba conculcada en Chile dado el contexto dictatorial de la época, rompiendo la histórica tradición sectorial de colaboración recíproca entre el Estado, los equipos de salud y la comunidad organizada, que buscaba incorporar a toda la población nacional en los beneficios de salud. ${ }^{14}$ Con el proceso de transición democrática iniciado en 1990, la participación es revalorizada desde instancias técnicas y políticas, expresándose en la decidida reactivación de los Consejos Locales de Salud, creados originalmente en el año 1971 por el Ministerio de Salud. ${ }^{15}$ Asimismo, a contar de 1992, se destinan recursos para la conformación de equipos de reforzamiento psicosocial, ${ }^{16}$ cuyas primeras tareas asignadas promovieron la participación ciudadana, con énfasis en la recomposición de los vínculos entre las organizaciones sociales y los equipos de salud, relación resentida por los largos años dictatoriales que impidieron la comunicación entre ambas instancias y cuyos efectos se amplificaban debido a las fuertes restricciones que había enfrentado la atención primaria a partir del proceso de municipalización.

Años más tarde, la Estrategia Nacional de Salud para el cumplimiento de los Objetivos Sanitarios de la Década, Periodo 2011-2020, ${ }^{17}$ definió la participación en salud como Objetivo Estratégico $\mathrm{N}^{\mathrm{0} 7}$-en el marco de nueve formulaciones estratégicas- robusteciendo el sistema público al aumentar los mecanismos de participación ciudadana. Se reconocieron sus aportes a las prácticas de autocuidado de la población, su efecto multiplicador en la promoción de la salud y su invaluable colaboración en acciones de apoyo con la población usuaria afectada por patologías crónicas, dismovilidad o enfermedades terminales, a través de agrupaciones comunitarias que amplifican las respuestas sanitarias y propician mejores 
soluciones a las expectativas de la población. El año 2012 se dicta la Ley № 20.584 que explícita los derechos y deberes de la población usuaria, considerando especialmente los relacionados con la dignidad, la autonomía y la información, como base para la participación en salud. ${ }^{18}$ Asimismo, el Ministerio de Salud ha formulado las orientaciones técnicas para la participación en atención primaria, definiéndola como "una relación de colaboración y respeto mutuo entre el Estado y la ciudadanía, que favorece el fortalecimiento de la sociedad civil y permite una mayor legitimidad y sustento ético de las políticas públicas". ${ }^{19}$ Consecuentemente, propone una tipología de participación según objetivos $^{20}$ que transita desde una menor hacia mayor complejidad, identificando los siguientes niveles: i) informativo, garantizando a la ciudadanía información y acceso al sistema de salud, expresada principalmente en las Oficinas de Información, Reclamos y Sugerencias (OIRS); ii) consultivo sobre decisiones en materia de políticas públicas sanitarias, en carácter vinculante o no vinculante, a través de consultas o diálogos ciudadanos; iii) gestionaria, activando las capacidades de las personas para la movilización y gestión de recursos en favor de objetivos comunes, como ocurre en la planificación local participativa; iv) habilitadora social, identificando problemas comunes y buscando soluciones en forma organizada a través de diagnósticos participativos locales; y v) empoderamiento, expresado en la capacidad de incidencia de la ciudadanía en la toma de decisiones en políticas y acciones que les afectan, como sucede en los presupuestos ciudadanos. La participación representa un espacio efectivo de incorporación de las organizaciones comunitarias en las estrategias orientadas a intervenciones centradas en la salud y a intervenciones centradas en la enfermedad, complementariedad virtuosa de la acción sanitaria propuesta con visión estratégica hace 40 años en la Declaración de Alma Ata.

3. Intersectorialidad. Constituye una oportunidad de movilización integrada de recursos y de coordinación de acciones de diversos sectores de la sociedad, contribuyendo de manera directa en la mejora de la salud de la población, especialmente en las acciones de promoción, prevención y educación en salud. Su mayor expresión es la Comisión Mixta Salud Educación COMSE, trabajo intersectorial que tiene su formalización en el año $1955,{ }^{21}$ a través de los Decretos Supremos № 48 del Ministerio de Salud Pública y Previsión Social y №12.735 del Ministerio de Educación Pública. Se buscaba la coordinación de las instituciones de salud y educacionales en un mismo territorio, considerando los equipos profesionales de ambos sectores, e integrando a la familia y a la comunidad en favor de la salud infantil. Esta instancia intersectorial se mantuvo en funciones durante los años dictatoriales, focalizada en acciones asistenciales, campañas de vacunación y labor educativa de bajo impacto orientada a grupos de interés. Esta instancia es revitalizada en democracia, a través del Decreto $N^{\circ} 585$ del Ministerio de Salud de 1996, que crea las Comisiones Mixtas Asesoras de Salud y Educación. ${ }^{22}$ Esta normativa dispuso la labor de asesoría y coordinación de las actividades conjuntas de los Ministerios de Salud y de Educación y sus organismos dependientes, en favor de dar cumplimiento a objetivos de interés común que consideren la educación sanitaria de la población infanto-juvenil. Este funcionamiento nacional se propone como replicable a nivel regional, provincial, comunal y local, siendo este último el único que se ha mantenido vigente a lo largo del tiempo, traducido en un trabajo colaborativo de cada Centro de Salud Familiar con los establecimientos educacionales de su territorio, desplegando acciones de carácter integral, que consideran la participación preferente de líderes o agentes comunitarios claves.

Otro importante esfuerzo intersectorial se desarrolla a contar del año 2007, con la creación del subsistema de protección integral Chile crece contigo, ${ }^{23}$ modelo de gestión intersectorial, en donde la atención primaria aporta desde su valiosa tradición materno infantil, en un conjunto de prestaciones orientadas a la población priorizada socialmente.

Actualmente, la intersectorialidad se expresa en relaciones de colaboración entre la atención primaria y los diversos organismos e instancias públicas y privadas con las que comparte 
su territorio. Estas relaciones se traducen en redes con municipios y servicios regionales de vivienda, urbanismo, previsión social y medio ambiente, entre los de mayor recurrencia. Contempla la coordinación con servicios de atención preferente a infancia, adolescencia, mujeres, migrantes, pueblos originarios y población adulta mayor y aporta en situaciones de catástrofe y emergencia a nivel local, regional o interregional. En estos contextos, se reconoce en los equipos de atención primaria la preeminencia de los roles de liderazgo técnico sanitario y de gestión de redes institucionales y comunitarias, permitiendo a través del trabajo colaborativo amplificar sus esfuerzos en la concreción del desafío impuesto por Alma Ata, para llevar la asistencia sanitaria lo más cerca posible del lugar donde residen y trabajan las personas.

\section{REFLEXIONES FINALES}

La Declaración de Alma Ata reconoció la condición de la Salud como un Derecho Humano para todos los pueblos y regiones del mundo, definiendo a la universalidad, la participación y la intersectorialidad como los criterios centrales para avanzar en su concreción. Desde este marco, la realidad sanitaria chilena sugiere que persisten significativas brechas en torno a estos referentes centrales. Así, entonces, en el criterio de universalidad se mantienen importantes inequidades estructurales en el acceso a la salud, relacionadas directamente con las condiciones socioeconómicas de la población y con la persistencia de dificultades en la accesibilidad y cobertura de la atención sanitaria oportuna en zonas geográficas de difícil acceso o alta ruralidad, afectando directamente el bienestar de la población. Asimismo, la progresiva complejización demográfica y epidemiológica nacional ha mantenido a la atención primaria chilena en condiciones de sobreexigencia permanente frente a los desafíos derivados por una parte, de la mantención de los avances sanitarios alcanzados en décadas pasadas y por otra, de la urgente renovación de sus líneas de trabajo demandada por la emergencia de nuevos fenómenos sanitarios contemporáneos ligados principalmente al envejecimiento de la población y a los procesos migratorios.
Respecto de la capacidad resolutiva de la atención primaria en forma específica, aún es frecuente observar ocasiones en que es sobrepasada operativamente, especialmente en comunas cuya población pertenece mayoritariamente a sectores de bajos ingresos, población envejecida, población en tránsito por razones de migración o por acceso a ciclos estacionales de empleo; junto con la sobredemanda derivada de los períodos invernales o por emergencias sanitarias que incrementan la solicitud de servicios por atención directa y por derivación por interconsulta a atenciones de mayor complejidad. Asimismo, se advierten efectos adversos como resultado de ciclos recesivos de la economía nacional, que ocasionan cesantía y pérdida de plazas laborales a parte de la población. En esas ocasiones, la pérdida de cobertura de los sistemas de protección social privados, redirecciona la demanda de atención sanitaria a la salud pública, siendo su primera línea de contención la atención primaria local.

También debe tenerse presente que las garantías de cobertura y atención oportuna incorporados explícitamente por el GES en un conjunto de prestaciones de salud, han sumado un nuevo frente de tensión. Lo anterior, especialmente cuando los recursos disponibles no permiten garantizar el cumplimento pleno de los compromisos declarados desde la propia capacidad resolutiva instalada en la salud pública, forzando la compra de servicios complementarios en el extra sistema, en donde la preeminencia de las lógicas de mercado conspiran contra la concepción de la salud como un Derecho Humano garantizado por el Estado, afectando fuertemente los presupuestos sectoriales.

Respecto del criterio de participación en salud, se considera que continúa plenamente vigente a partir de los referentes de Alma Ata, incorporando su valioso aporte en el análisis participativo de las necesidades sanitarias y en la toma de decisiones pertinentes en salud, permitiendo reflejar con precisión las prioridades y urgencias definidas desde la población. No obstante, debe reconocerse que actualmente se presentan importantes dificultades en este criterio, asociadas al desdibujamiento de la 
concepción de la salud como un derecho social y una responsabilidad compartida, la que ha sido reemplazada por la valorización de la salud como un bien al que se accede en condiciones de mercado. Como consecuencia, la participación comunitaria se ha resignificado desde una posición protagónica inicial de sujeto de derechos y deberes, a una posición de población usuaria que demanda prestaciones en los servicios sanitarios en calidad de cliente, desdibujando la comprensión de la salud como una aspiración de accesibilidad, equidad y calidad de servicios socialmente compartida.

No obstante, debe mencionarse que la excepción a esta situación la constituye la población adulta mayor, heredera de una tradición de participación social forjada en las décadas de 1950 y 1960, en donde la atención primaria de salud les incorporó activamente como actores sociales claves para apoyar la ampliación de su infraestructura, cobertura, programas y servicios. Esta situación de colaboración mutua fue interrumpida de manera traumática durante el período 1973-1990 y posterior a esa fecha, ha sido la normalización del funcionamiento democrático del país la que ha permitido reactivar los aprendizajes organizacionales pretéritos de dicha población, aportando con su valioso compromiso en el desarrollo de las actuales experiencias de participación comunitaria en salud, a través de Consejos Locales de Salud, clubes de adultos mayores, actividades de educación en salud y funcionamiento regular de grupos de salud mental, que permiten ilustrar las instancias de mayor adhesión de este grupo social a nivel primario.

En relación a la intersectorialidad, puede precisarse que sigue siendo un criterio deseable que se realiza con mayor dinamismo a nivel territorial local, perdiendo impacto en niveles comunales, regionales y nacionales. La experiencia indica que su dificultad central se manifiesta en la limitada capacidad de convocatoria de instancias representativas del sector privado en torno a las tareas sanitarias mancomunadas, por lo que los actuales esfuerzos intersectoriales se expresan principalmente a través del compromiso con las acciones de salud primarias que realiza el sector público, por medio de ministerios, secretarías regionales y municipios, con el apoyo complementario de organizaciones sociales y movimientos ciudadanos.

Reconociendo que aún persisten brechas respecto de los postulados centrales de Alma Ata, debe valorarse que la atención primaria chilena ha hecho avances significativos en las últimas décadas, producto de un esfuerzo nacional realizado en forma conjunta por las autoridades sectoriales, los equipos de salud primaria, la academia, el intersector y la comunidad. La definición de la Salud como un Derecho Humano fundamental se mantiene como una legítima aspiración social que enfrenta permanentemente las dificultades derivadas de las condiciones estructurales de funcionamiento legal, administrativo y de financiamiento del sistema de salud chileno, contribuyendo a que persistan déficit históricos y se sumen nuevas demandas insatisfechas. A pesar de lo anterior, puede declararse como corolario que la estrategia de desarrollo que representa la atención primaria y sus criterios de universalidad, participación e intersectorialidad siguen plenamente vigentes, siendo respaldados por el renovado compromiso país de continuar en sus esfuerzos por alcanzar en un futuro próximo la promesa de Salud para Todos propuesta por Alma Ata en 1978. 


\section{REFERENCIAS BIBLIOGRÁFICAS}

1. Oficina Panamericana de la Salud. Declaración de Alma Ata. Conferencia Internacional sobre Atención Primaria de Salud, Alma-Ata, URSS, 6-12 Septiembre 1978. [internet] OPS; citado 15 mayo 2018]. Disponible en https://www. paho.org/hq/index.php?option=com docman\&task=doc_view\&gid=19004\&Item id $=2518$ \&lang $=$ en

2. López M. ¿Salud para todos? La atención primaria de salud en Chile y los 40 años de Alma Ata, 1978-2018. [internet]. Santiago, Chile: Ministerio de Salud, 2018. Citado 05 mayo 2018] Disponible en http://www.repositoriodigital.minsal.cl/ handle/2015/418

3. República de Chile. Decreto con Fuerza de Ley № 1-3 que Reglamenta Aplicación del Inciso Tercero del Artículo 38 del Decreto Ley $\mathrm{N}^{\circ} 3.063$ de 1979. [internet] Ministerio del Interior, 1980 [citado el 16 mayo 2018] Disponible en http://bcn.cl/1 vhvk

4. MINSAL. Financiamiento de la Atención Primaria de Salud Municipal. Evaluación de indexadores per cápita basal. [internet] Santiago de Chile. Gobierno de Chile, Ministerio de Salud, 2012. Citado 07 mayo 2018] Disponible en http://www. bibliotecaminsal.cl/wp/wp-content/ uploads/2018/01/001. Financiamientode-la-Atenci \%C3\%B3n-Primaria-deSalud-Municipal-evaluaci\%C3\%B3n-deindexadores-del-per-capita-basal.pdf

5. Sacarpaci J. Salud y Régimen Militar. Santiago de Chile. Colectivo de Atención Primaria; 1988.

6. MINSAL. Manual administrativo para servicios de atención primaria de urgencia. Subsecretaria Redes Asistenciales. División Gestión de la Red Asistencial. Serie Cuadernos Manual Administrativo No6. [internet] Santiago de Chile. Ministerio de Salud, 2007. Citado 07 mayo 2018] Disponible en http://www.bibliotecaminsal. cl/wp/wp-content/uploads/2016/03/6.pdf

7. MINSAL. Modelo Nacional Sistema de Atención Médica de Urgencia SAMU. Departamento de Gestión del Riesgo
Asistencial. División de Gestión de Redes Asistenciales. Subsecretaría de Redes Asistenciales. [internet] Santiago de Chile. Ministerio de Salud, 2018. Citado 09 mayo2018] Disponible en: http://www.minsal. cl/wp-content/uploads/2018/03/ModeloNacional-Sistema-de-Atenci\%C3\%B3nM\%C3\%A9dica-de-Urgencia-SAMU.pdf

8. MINSAL. Programa Servicio de Urgencia Rural SUR. Subsecretaría de Redes Asistenciales. División de Atención Primaria. [internet] Santiago de Chile. Ministerio de Salud, 2014. citado 09 mayo 2018] Disponible en https:// www.araucanianorte.cl/images/PDF-WORD/ Resolucion-1158-programa-servicio-deurgencia-rural-sur.pdf

9. República de Chile. Ley $N^{\circ} 19.378$ que establece Estatuto de Atención Primaria de Salud Municipal. [internet] Santiago de Chile. Ministerio de Salud, 1995. Citado 10 Mayo 2018] Disponible en www.bcn.cl/ historiadelaley/nc/historia-de-la-ley/7114/

10. MINSAL. Red de Servicios de Salud Mental y Psiquiatría. [internet) Santiago de Chile. Ministerio de Salud, 2000. Citado 14 mayo 2018]. Disponible en http://www.minsal.cl/portal/url/ item/71e4f2dd2b638460e04001011f01239d. pdf

11. República de Chile. Ley No 19.966 que establece un Régimen de Garantías en Salud. [internet] Santiago de Chile. Ministerio de Salud, 2008. Citado 10 mayo 2018] Disponible en http://bcn.cl/1 uyp6

12. MINSAL. Orientaciones para la implementación del modelo de atención integral de salud familiar y comunitaria. [internet] Santiago de Chile. Subsecretaría de Redes Asistenciales. División de Atención Primaria, 2012. Citado 07 mayo 2018] Disponible en http://www.minsal.cl/portal/url/ item/e7b24eef3e5cb5d1e0400101650128e9. pdf

13. MINSAL. Plan para Pueblos Indígenas. Proyecto de Apoyo al Sector Salud MINSAL - Banco Mundial [internet] Santiago de Chile, Ministerio de Salud, 2017. Citado 14 mayo 2018]. Disponible en http://www.minsal.cl/ wp-content/uploads/2017/04/PPI-_Chile_ HESSP-7-de-abril-2017-MINSAL-OK.pdf 
14. Castañeda P. y Salamé A.M. Trabajo Social chileno y dictadura militar. Memoria Profesional predictatorial. Participación Social en Salud. Período 1960-1973. Rev. Perspectivas [internet] 2016 [citado 15 mayo 2018] 27 (2): 29-57. Disponible en http://ediciones.ucsh.cl/ojs/index.php/ Perspectivas/article/download/410/360

15. República de Chile. Decreto 602 que crea Consejos Locales de Salud. [internet] Santiago de Chile Ministerio de Salud, 1971 [citado 12 mayo 2018] Disponible en http:// bcn.cl/21a2l

16. MINSAL. Políticas y Plan de Salud Mental. Santiago de Chile, Ministerio de Salud; 1993.

17. MINSAL. Estrategia Nacional de Salud para el cumplimiento de los Objetivos Sanitarios de la década 2011-2020. [internet] Santiago de Chile. Ministerio de Salud, 2011. Citado 09 mayo 2018] Disponible en http://www.minsal.cl/portal/url/item/ c4034eddbc96ca6de0400101640159b8.pdf

18. República de Chile. Ley $\mathrm{N}^{\circ} 20.584$. Regula los derechos y deberes que tienen las personas en relación con acciones vinculadas a su atención en salud. [internet] Santiago de Chile. Ministerio de Salud, 2012. Citado 11 mayo 2018] Disponible en http://bcn.cl/1uw7l

19. MINSAL. Orientaciones para la planificación y programación en red. [internet]. Santiago de Chile. Ministerio de Salud. Subsecretaría de Redes Asistenciales, 2018. Citado 11 mayo 2018]. Disponible en http://www. minsal.cl/wp-content/uploads/2015/10/ Orientaciones-para-la-planificacion-yprogramacion-en-red-2018.pdf

20. MINSAL. Participación ciudadana en Atención Primaria. Un aporte del Modelo de Salud Familiar y Comunitaria a la satisfacción usuaria. Santiago de Chile. [internet] Ministerio de Salud. Subsecretaría de Redes Asistenciales. División de Atención Primaria, 2012; citado 10 mayo 2018] Disponible en http://www.minsal.cl/wp-content/ uploads/2016/09/2_PARTICIPACI\%C3\%93NCIUDADANA-APS.pdf

21. SNS. Boletín $N^{\circ}$ 2. Santiago de Chile. Servicio Nacional de Salud [internet] 1956 [citado 11 mayo 2018] Vol 2 (2): 39-44 Disponible en http://www.bibliotecaminsal. cl/wp/wp-content/uploads/2013/08/Boletinmar-abr-1956.pdf

22. MINSAL. Decreto 585. Crea Comisiones Mixtas Asesoras de Salud y Educación. Santiago de Chile. Ministerio de Salud, 1997 [internet]; citado 09 mayo 2018] Disponible en http://bcn.cl/206an

23. MIDEPLAN. Ley $\mathrm{N}^{\circ} 20.379$ Crea el sistema intersectorial de protección social e institucionaliza el subsistema de protección integral a la infancia "Chile crece contigo". Santiago de Chile. Ministerio de Planificación, 2009. [internet]; citado 10 mayo 2018] Disponible en http://bcn. cl/1uuz0 